\title{
FULL FLOW/MOTION-FIELD RECOVERY FROM PULSED-WAVE ULTRASOUND DOPPLER DATA
}

\author{
Muthuvel Arigovindan, Michael Sühling*, Christian Jansen*, Patrick Hunziker ${ }^{*}$, and Michael Unser \\ École polytechnique fédérale de Lausanne (EPFL) \\ Biomedical Imaging Group \\ CH-1015 Lausanne, Switzerland \\ E-mail of corresponding author: michael.unser@epfl.ch
}

\section{ABSTRACT}

We present a new computational method for reconstructing a vector field from scattered, pulsed-wave ultrasound Doppler data. The main difficulty is that the Doppler measurements are incomplete, for they do only capture the velocity component along the beam direction. We thus propose to combine measurements from different beam directions. However, this is not yet sufficient to make the problem well posed because (i) the angle between the directions is typically small; and (ii) the data is noisy and nonuniformly sampled. We therefore introduce a vector-spline regularization that forces the solution to be well behaved while inducing some helpful coupling between the $x$ and $y$ components of the vector field. We discretize our vector-spline reconstruction problem in a uniform B-spline basis, obtaining a sparse system of equations that can be solved efficiently. We validate our method using real tissue Doppler data for which the ground truth is known. Finally, we present some patient results obtained with color Doppler imaging; in particular, we are able to observe bloodflow variations in the bifurcation of the carotid artery.

\section{INTRODUCTION}

Pulsed-wave ultrasound Doppler (PWD) imaging is an effective tool for the monitoring of vascular and cardiac function $[1,2,3]$. The imaging system sends a periodic pulse train along a set of scan lines and measures the backscattered signal. By analyzing the Doppler frequency shift in the received signal, the system retrieves a set of axial velocity estimates; these are the projected components of the true 3-D velocity along the direction of the ultrasound beam. These axial components are resampled on a regular grid and presented in a color-coded form that is known as the color Doppler image.

*M. Sühling is with Siemens AG, Allee am Röthelheimpark 2, D-91052 Erlangen, Germany, e-mail: michael.suehling@siemens.com. C. Jansen and P. Hunziker are with the Kantonsspital Basel, Petersgraben 4, CH-4031 Basel, e-mail: christian.jansen@unibas.ch, phunziker@uhbs.ch.

This work was supported in part by the Swiss Heart Foundation and the Swiss National Science Foundation under grant 200020-109415.
The color flow image sequence contains the instantaneous velocity information, but it is incomplete; in particular, the system is blind to the motion that is orthogonal to the beam. Nevertheless, such a partial velocity field has been used for the determination of quantitative parameters such as flow volume. Further, the qualitative, and, to some extent, the quantitative motion analysis obtained from this kind of data was found to be clinically useful in several instances [2, 3]. However, the availability of a full vector field is desirable since it will lead to a more flexible quantitative analysis.

In this paper, we propose a method to reconstruct the true velocity field from the PWD data. We formulate the task as the problem of finding a continuous vector function that is the minimizer of a suitable criterion. Specifically, we consider a quadratic-cost functional that is a weighted sum of a data term, along with a physically inspired smoothness functional (regularizer). The regularizer enforces some coupling between the $x$ and $y$ velocity components. We demonstrate that the partial nature of the data makes this coupling crucial. We search for the continuous solution in a shift-invariant space. By choosing the step size, we can control the tradeoff between computational complexity and reconstruction accuracy.

We validate our method using a real phantom experiment. Finally, we provide some reconstruction results on blood-pool data; specifically, we reconstruct the blood flow field in the carotid bifurcation.

\section{PROPOSED RECONSTRUCTION METHOD}

\subsection{Form of the Data}

A Doppler imaging system measures axial velocities at some selected locations in the cross-sectional plane under consideration. The selected locations $\left\{\mathbf{x}_{i}\right\}$ are typically located on a noncartesian grid. These nonuniform measurements are scanconverted (resampled in a regular grid) and then displayed in a color-coded form.

A typical 2-D data set will contain the set of sampling locations $\left\{\mathbf{x}_{i}\right\}$, the corresponding beam directions $\left\{\mathbf{d}_{i}\right\}$, and 
the Doppler measurements $\left\{m_{i}\right\}$ satisfying $m_{i}=\mathbf{d}_{i}^{T} \mathbf{v}\left(\mathbf{x}_{i}\right)$, where $\mathbf{v}(\mathbf{x})$ is the true velocity field. Note that the sampling locations $\left\{\mathbf{x}_{i}\right\}$ are nonuniform, especially when there are multiple views.

Figure 1 gives the schematic of two commonly used sampling geometries. The solid lines with arrows represent the scan lines (ultrasound beam), whereas the dotted lines represent the sampling grid along the scan lines. In the cone-beam or sector-beam scheme, the beams are launched from a single point along a series of regularly spaced angles. In the parallel-beam acquisition, beams are launched with a fixed angle from a set of points spaced regularly along a line. A typical parallel-beam probe has the capability of steering the beam angle, which means that different Doppler images can be acquired without moving the probe (multiple acquisitions).
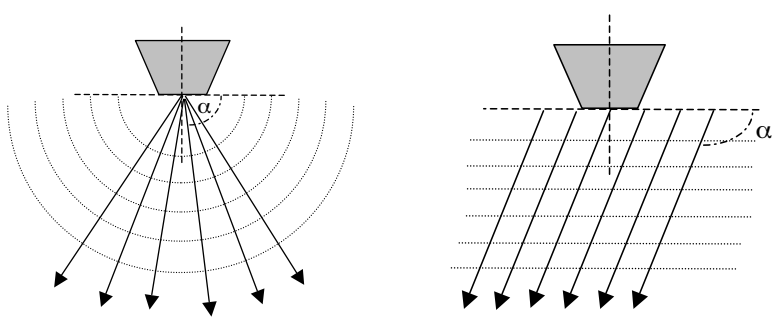

(a) Cone-beam or sector-beam acquisition

(b) Parallel-beam acquisition

Fig. 1. Scan-line schemes for 2-D acquisition

In this paper, we consider two types of data sets: (i) samples of multiple acquisitions of the same cross-sectional plane obtained from a cone-beam probe placed at different locations (sample locations and beam directions are transformed into a common reference plane); (ii) multiple acquisitions from a parallel-beam probe obtained by beam steering.

\subsection{Proposed Formulation}

Let $\mathbf{v}(\mathbf{x})=[u(x, y) v(x, y)]$ be the velocity field. Our aim is to recover $\mathbf{v}(\mathbf{x})$ from the given Doppler measurement set $\left\{\mathbf{x}_{i}, \mathbf{d}_{i}, m_{i}\right\}$. We search for the solution in the space of uniform B-splines; in other words, we restrict the velocity field to be of the following form:

$$
\begin{aligned}
& u(x, y)=\sum_{k=0}^{N_{g}-1} \sum_{l=0}^{N_{g}-1} c_{k, l}^{u} \beta^{n}(x / a-k) \beta^{n}(y / a-l), \\
& v(x, y)=\sum_{k=0}^{N_{g}-1} \sum_{l=0}^{N_{g}-1} c_{k, l}^{v} \beta^{n}(x / a-k) \beta^{n}(y / a-l) .
\end{aligned}
$$

Here, $\beta^{n}$ is the B-spline of degree $n$ and $a$ is a step size that controls the accuracy. $N_{g}$ is the grid size. The idea is to formulate the reconstruction as the minimizer of a quadraticcost functional.

The cost to minimize is

$$
J_{V R L S}(\mathbf{v})=\sum_{i=1}^{N}\left(\mathbf{d}_{i}^{T} \mathbf{v}\left(\mathbf{x}_{i}\right)-m_{i}\right)^{2}+\lambda R_{E}(\mathbf{v})
$$

where $R_{E}(\mathbf{v})$ is our specific choice of smoothness functional which involves the divergence and the curl of the velocity field, and which is computed as follows:

$$
\begin{aligned}
& R_{E}(\mathbf{v})=\lambda_{d_{0}} \int(\operatorname{div} \mathbf{v}(\mathbf{x}))^{2} d \mathbf{x}+\lambda_{c_{0}} \int(\operatorname{curl} \mathbf{v}(\mathbf{x}))^{2} d \mathbf{x} \\
& +\lambda_{d_{1}} \int\|\nabla(\operatorname{div} \mathbf{v}(\mathbf{x}))\|^{2} d \mathbf{x}+\lambda_{c_{1}} \int\|\nabla(\operatorname{curl} \mathbf{v}(\mathbf{x}))\|^{2} d \mathbf{x} .
\end{aligned}
$$

Since the divergence gives the density change, the first term in (4) quantifies the overall compression rate, whereas the second term gives its spatial roughness. Both terms are related to the deformation of the medium. The third term sums up the squared angular velocity. It does not directly quantify the deformation, but will tend to penalize rotations_-including rigid ones. The last term, on the other hand, is indeed a measure of deformation, as it captures the spatial variation of the angular velocity.

The idea behind using this regularizer is that it includes every derivative-based quantity that has a direct physical interpretation. This allows one to incorporate some a priori knowledge on the type of velocity field, and also to specify physically plausible solutions. Interestingly, we have verified that any rotationally-invariant functional of order less or equal to two takes the form (4). The proof will be published elsewhere.

An important point is that the regularizer has cross terms that induce a coupling between the vector-field components. Yet, the present functional also includes standard, uncoupled regularizers as special cases. Specifically, when $\lambda_{d_{0}}=\lambda_{c_{0}}>$ 0 and $\lambda_{d_{1}}=\lambda_{c_{1}}=0$, (4) becomes equivalent to an independent gradient regularizer (membrane spline) on each component separately. Likewise, when $\lambda_{d_{1}}=\lambda_{c_{1}}>0$ and $\lambda_{d_{0}}=$ $\lambda_{c_{0}}=0$, (4) becomes equivalent to an independent Laplacian regularizer (thin-plate spline [4]) on each component separately. Moreover, choosing $\lambda_{d_{0}}=\lambda_{c_{0}}>0$ and $\lambda_{d_{1}}=\lambda_{c_{1}}>$ 0 yields the so-called splines under tension, which involve a weighted sum of Duchon's seminorms [5]. The interesting aspect of these (uncoupled) functionals is that the corresponding nonparametric regularized least-squares approximation problem can be solved analytically and its solution expressed as a sum of radial-basis functions [6].

In contrast with the works cited above, we argue here that the presence of coupling is crucial for our application; this is also supported by our experimental results. 


\subsection{Solution}

Let $\mathbf{c}_{u}=\left[\cdots c_{k, l}^{u} \cdots\right]^{T}, \mathbf{c}_{v}=\left[\cdots c_{k, l}^{v} \cdots\right]^{T}$, and $\mathbf{c}=\left[\mathbf{c}_{u}^{T} \mathbf{c}_{v}^{T}\right]^{T}$. The required coefficient vector that minimizes (3) is the solution of the following equation:

$$
\mathbf{A c}=\mathbf{b}
$$

where

$$
\begin{aligned}
\mathbf{A} & =\left[\begin{array}{cc}
\mathbf{D}_{u} & \mathbf{D}_{u v} \\
\mathbf{D}_{u v} & \mathbf{D}_{v}
\end{array}\right]+\left[\begin{array}{cc}
\mathbf{R}_{u} & \mathbf{R}_{u v} \\
\mathbf{R}_{u v} & \mathbf{R}_{v}
\end{array}\right] \\
\mathbf{b} & =\left[\begin{array}{l}
\mathbf{b}_{u} \\
\mathbf{b}_{u}
\end{array}\right] .
\end{aligned}
$$

The submatrices are given by

$$
\begin{aligned}
& \left\{\mathbf{D}_{u}\right\}_{N_{g} k+j, N_{g} m+l}=\sum_{i}\left(d_{x i}^{2} \beta^{n}\left(x_{i} / a-j\right) \beta^{n}\left(y_{i} / a-k\right)\right. \\
& \left.\times \beta^{n}\left(x_{i} / a-l\right) \beta^{n}\left(y_{i} / a-m\right)\right), \\
& \left\{\mathbf{D}_{v}\right\}_{N_{g} k+j, N_{g} m+l}=\sum_{i}\left(d_{y i}^{2} \beta^{n}\left(x_{i} / a-j\right) \beta^{n}\left(y_{i} / a-k\right)\right. \\
& \left.\times \beta^{n}\left(x_{i} / a-l\right) \beta^{n}\left(y_{i} / a-m\right)\right),
\end{aligned}
$$$$
\left\{\mathbf{D}_{u v}\right\}_{N_{g} k+j, N_{g} m+l}=\sum_{i}\left(d_{x i} d_{y i} \beta^{n}\left(x_{i} / a-j\right) \beta^{n}\left(y_{i} / a-k\right)\right.
$$$$
\left.\times \beta^{n}\left(x_{i} / a-l\right) \beta^{n}\left(y_{i} / a-m\right)\right) .
$$

The matrices $\mathbf{R}_{u}, \mathbf{R}_{v}$, and $\mathbf{R}_{u v}$, are the circulant matrices corresponding to the filters $R_{u}\left(z_{1}, z_{2}\right), R_{u}\left(z_{1}, z_{2}\right)$, and $R_{u v}\left(z_{1}, z_{2}\right)$, respectively. These are given by

$$
\begin{aligned}
R_{u}\left(z_{1}, z_{2}\right) & =\lambda_{d_{0}} R_{11}\left(z_{1}, z_{2}\right)+\lambda_{c_{0}} R_{12}\left(z_{1}, z_{2}\right), \\
& +\lambda_{d_{1}} R_{21}\left(z_{1}, z_{2}\right)+\lambda_{c_{1}} R_{22}\left(z_{1}, z_{2}\right) \\
R_{v}\left(z_{1}, z_{2}\right) & =\lambda_{d_{0}} R_{12}\left(z_{1}, z_{2}\right)+\lambda_{c_{0}} R_{11}\left(z_{1}, z_{2}\right), \\
& +\lambda_{d_{1}} R_{22}\left(z_{1}, z_{2}\right)+\lambda_{c_{1}} R_{21}\left(z_{1}, z_{2}\right) \\
R_{u v}\left(z_{1}, z_{2}\right) & =\left(\lambda_{d_{0}}-\lambda_{c_{0}}\right) R_{13}\left(z_{1}, z_{2}\right) \\
& +\left(\lambda_{d_{1}}-\lambda_{c_{1}}\right) R_{23}\left(z_{1}, z_{2}\right),
\end{aligned}
$$

where

$$
\begin{aligned}
R_{11}\left(z_{1}, z_{2}\right) & =L\left(z_{1}\right) B^{2 n-1}\left(z_{1}\right) B^{2 n+1}\left(z_{2}\right), \\
R_{12}\left(z_{1}, z_{2}\right) & =B^{2 n+1}\left(z_{1}\right) L\left(z_{2}\right) B^{2 n-1}\left(z_{2}\right), \\
R_{13}\left(z_{1}, z_{2}\right) & =B^{\prime 2 n+1}\left(z_{1}\right) B^{\prime 2 n+1}\left(z_{2}^{-1}\right), \\
R_{21}\left(z_{1}, z_{2}\right) & =\frac{1}{a^{2}}\left[\left(L\left(z_{1}\right)\right)^{2} B^{2 n-3}\left(z_{1}\right) B^{2 n+1}\left(z_{2}\right)\right. \\
& \left.+L\left(z_{1}\right) B^{2 n-1}\left(z_{1}\right) L\left(z_{2}\right) B^{2 n-1}\left(z_{2}\right)\right](13) \\
R_{22}\left(z_{1}, z_{2}\right) & =\frac{1}{a^{2}}\left[B^{2 n+1}\left(z_{1}\right)\left(L\left(z_{2}\right)\right)^{2} B^{2 n-3}\left(z_{2}\right)\right. \\
& \left.+L\left(z_{1}\right) B^{2 n-1}\left(z_{1}\right) L\left(z_{2}\right) B^{2 n-1}\left(z_{2}\right)\right](14) \\
R_{23}\left(z_{1}, z_{2}\right) & =\frac{1}{a^{2}}\left[L\left(z_{1}\right) D^{2 n-2}\left(z_{1}\right) B^{\prime 2 n+1}\left(z_{2}^{-1}\right)\right. \\
& \left.+D^{2 n+1}\left(z_{1}\right) L\left(z_{2}\right) B^{\prime 2 n-2}\left(z_{2}^{-1}\right)\right],
\end{aligned}
$$

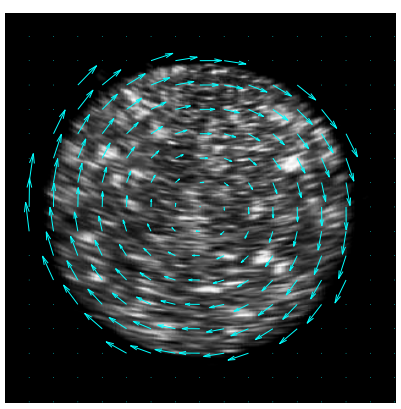

Fig. 2. Reconstructed motion field for the rotating real phantom.

with $B^{n}(z)$ being the discrete B-spline of degree $n$ and with $L(z)=z+2+z^{-1}$. The subvectors $\mathbf{b}_{u}$ and $\mathbf{b}_{u}$ are given by

$$
\begin{aligned}
\left\{\mathbf{b}_{u}\right\}_{N_{g} k+j} & =\sum_{i} d_{x i} \beta^{n}\left(x_{i} / a-j\right) \beta^{n}\left(y_{i} / a-k\right), \\
\left\{\mathbf{b}_{v}\right\}_{N_{g} k+j} & =\sum_{i} d_{y i} \beta^{n}\left(x_{i} / a-j\right) \beta^{n}\left(y_{i} / a-k\right) .
\end{aligned}
$$

The linear system above is sparse, well conditioned, and diagonally dominant. Thanks to these properties, we obtain an efficient numerical implementation using standard sparsematrix routines. This is the primary advantage of the proposed method over a direct analytical solution (generalized thin-plate splines) which should also be feasible, in the spirit of the work of Suter al. [6].

\section{EXPERIMENTS}

\subsection{Real Phantom Experiment}

The real phantom that we constructed for this experiment is a cylindrical tissue-mimicking object (sponge) immersed in a water container. The object rotates with a constant angular velocity. Doppler data were acquired, and reconstruction was performed from two views differing by 10 degrees. Figure 2 shows one frame of the B-mode intensity image with the superimposed reconstructed motion field. The input SNR (on the projected velocities) is $13 \mathrm{~dB}$. The reconstruction SNR (full vector field) is $13.67 \mathrm{~dB}$. The preferred settings for the regularization parameters is $\left\{\lambda_{d_{0}} \rightarrow \infty, \lambda_{d_{1}}=0, \lambda_{c_{0}}=\right.$ $\left.0, \lambda_{c_{1}}=k \sigma^{2}\right\}$, where $\sigma^{2}$ is the noise variance and $k$ is a proportionality constant. We also observed that enforcing $\lambda_{d_{0}}=\lambda_{c_{0}}$ and $\lambda_{d_{1}}=\lambda_{c_{1}}$ (uncoupled regularization) degrades the performance by $5 \mathrm{~dB}$ or more.

\subsection{Blood Flow in the Carotid Bifurcation}

Blood-flow patterns in the carotid bifurcation have been reported to have a significant influence on the development of atherosclerosis [7] which is a leading cause of heart attack and stroke. In this experiment, we attempted to reconstruct 
the complete blood-flow distribution in the carotid bifurcation from multiple-view Doppler data acquired using a parallelbeam probe setup with steering capability. We performed three acquisitions with beam angles $\alpha=70^{\circ}, 90^{\circ}, 110^{\circ}$. We extracted the frames that correspond to R-peak over two cycles. The noise variance of the Doppler data was estimated by comparing frames corresponding to different cycles. It was estimated to be $9 \%$ of the signal energy.

We used $75 \%$ (randomly selected) of the total measurements (Data Set A) for reconstruction, and the remaining samples (Data Set B) for validation. In order to fine-tune the algorithm, we performed multiple reconstructions, adjusting the regularization parameters iteratively such that the data term of the cost functional matches the noise variance. At the end of the iterative procedure, the resulting data error was exactly $9 \%$ of the signal energy. Figure 3 presents the reconstruction results obtained from data set A. Figure 3(a) gives a visualization of the reconstructed flow field, whereas Figure 3(b) gives the flow profiles across the vessels before and after the bifurcation. We observe that, in the flow profiles after bifurcation, the maximum velocity is shifted towards the interior of the bifurcation, whereas in the flow profile before bifurcation, the maximum velocity stays approximately in the center of the vessel. This agrees with model-based predictions [8], and constitutes a significant clinical finding.

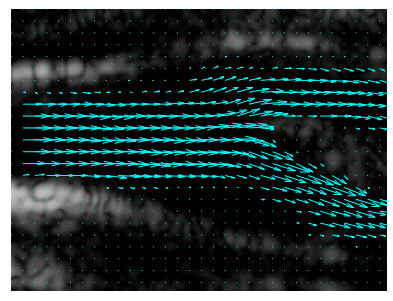

(a) Reconstructed flow field

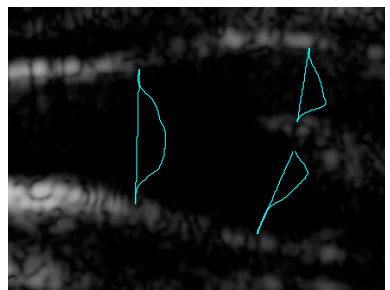

(b) Velocity profile across different parts of the vessels
Fig. 3. Reconstructed results for the carotid bifurcation

Next, we cross-validated the reconstructed flow field using Data Set B. Specifically, we synthesized the Doppler projections from the reconstructed flow field at the locations corresponding to Data Set $\mathrm{B}$, and compared them with the available data. The mismatch was found to be exactly $9 \%$ of the total energy. This indicates that the chosen values for the regularization parameters are matched to the data, and that the reconstructed velocity must be close to the underlying true flow field.

\section{CONCLUSION}

We demonstrated the feasibility of recovering true velocity fields from pulsed-wave Doppler data. We adopted a regularized least-squares approach, where the cost to be minimized is a weighted sum of the error in the data and a suitable smoothness functional. We proposed a derivative-based smoothness functional that allows one to incorporate a priori knowledge about the type of motion and to penalize undesirable solutions. We proposed a continuous B-spline solution for this minimization problem, and showed how it could be determined by solving a system of linear equations. We validated the proposed method with real experimental data and demonstrated its potential for clinical investigation.

\section{REFERENCES}

[1] D. Evans and W. McDicken, Doppler Ultrasound: Physics, Instrumentation and Signal Processing, Wiley, 2000.

[2] G.R. Sutherland, M.J. Steward, K.W.E. Grounstroem, C.M. Moran, A. Fleming, F.J. Guell-Peris, R.A. Riemersma, L.N. Fenn, K.A. Fox, and W.N. Mc Dicken, "Color Doppler myocardial imaging: A new technique for assessment of myocardial function," J. Am. Soc. Echocardiogr., vol. 7, no. 5, pp. 441-458, Sept.-Oct. 1994.

[3] P.R. Hunziker, M.H. Picard, N. Jander, M. ScherrerCrosbie, M. Pfisterer, and P.T. Buser, "Regional wall motion assessment in stress echocardiography by tissue Doppler bull's-eyes," J. Am. Soc. Echocardiography, vol. 12, no. 3, pp. 196-202, Mar. 1999.

[4] F. L. Bookstein, Morphometric Tools for Landmark Data, Cambridge University Press, 1991.

[5] J. Duchon, "Splines minimizing rotation-invariant seminorms in Sobolev spaces," Constructive Theory of Functions of Several Variables, pp. 85-100, 1977.

[6] D. Suter and F. Chen, "Left ventricular motion reconstruction based on elastic vector splines," IEEE Transactions on Medical Imaging, vol. 19, pp. 295-305, 2000.

[7] DN. Ku, DP. Giddens, CK. Zarins, and S. Glagov, "Pulsatile flow and atherosclerosis in the human carotid bifurcation-Positive correlation between plaque location and oscillating shear stress," Atherosclerosis, pp. 293-302, 1985.

[8] DN. Ku, "Blood flow in arteries," Annual reviews of fluid mechanics, pp. 399-434, 1991. 